\title{
Systemic treatment of metastatic breast cancer: SABCS 2018
}

\author{
Theresa Westphal · Simon Peter Gampenrieder · Richard Greil
}

Received: 16 May 2019 / Accepted: 25 July 2019 / Published online: 15 August 2019

(C) The Author(s) 2019

\begin{abstract}
Summary This review summarizes clinically relevant trials in metastatic breast cancer (MBC) presented at the 2018 San Antonio Breast Cancer Symposium (SABCS). At the meeting updates of two large phase III studies were presented: (1) the biomarker analysis of IMpassion130, trying to define the target population for the checkpoint inhibitor atezolizumab in triple-negative MBC and (2) a subgroup analysis of SOLAR-1, where the efficacy and safety of the alpha-specific Phosphoinositide 3(PI3)-kinase inhibitor alpelisib in combination with fulvestrant was investigated in patients with hormone receptor positive (HR+), human epidermal growth factor receptor 2 negative (HER2-) MBC. Furthermore, we review the results of a randomized phase II trial (CCTG MA38) comparing a continuous daily dosing of palbociclib to the standard treatment dose, and the phase $1 \mathrm{~b}$ data of the B-cell lymphoma 2 (BCL-2) inhibitor venetoclax in combination with tamoxifen as a novel drug combination in patients with HR+/HER2-MBC.
\end{abstract}

Keywords San Antonio Breast Cancer Symposium • Highlights · Review · IMpassion130 - SOLAR-1

\section{Introduction}

At the 2018 San Antonio Breast Cancer Symposium (SABCS), no new data from large phase III clinical studies in metastatic breast cancer (MBC) were presented. In this short review we instead focus on a number of interesting smaller trials, ranging from early drug development to quality of life studies and supportive treatment trials. Those study results might have direct influence on daily practice or show strong signals for successful future drug development. In detail, we summarize updated results of two phase III trials (IMpassion130 and SOLAR-1) as well as two smaller trials in hormone-receptor (HR) positive, human epidermal growth factor receptor 2 (HER2)-negative MBC investigating two different dosing schedules of the cyclin-dependent kinase $4 / 6$ (CDK4/6) inhibitor palbociclib and a the combination of the BCL-2 inhibitor venetoclax with tamoxifen, respectively.

\section{IMpassion130: biomarker analysis}

The IMpassion130 study was a phase III trial that evaluated atezolizumab, an anti-PD-L1 antibody, in combination with nab-paclitaxel as first-line treatment for metastatic triple negative breast cancer (TNBC) [1]. In total, 902 patients were randomized 1:1 to nab-paclitaxel $100 \mathrm{mg} / \mathrm{m}^{2}$ i.v. (on days 1,8 and 15 of a 28-day cycle) plus atezolizumab $840 \mathrm{mg}$ i.v. every 2 weeks or nab-paclitaxel plus placebo.

The primary analysis was already presented at the ESMO congress 2018 and was published in the New England Journal of Medicine [2]. The study showed a significantly longer progression-free survival (PFS) by the addition of atezolizumab in the intentionto-treat (ITT) population (hazard ratio [HR] 0.80; 95\% confidence interval $[\mathrm{CI}] 0.69-0.92 ; P=0.002)$. 
In patients with $\mathrm{PD}-\mathrm{L} 1$ expression in at least $1 \%$ of tumor-infiltrating immune cells (PD-L1 IC+) the influence on PFS was more pronounced (HR 0.62; 95\%CI $0.49-0.78 ; P<0.001)$. At this time point, the interim overall survival (OS) analysis showed no statistical difference in the ITT population between the two study arms. Therefore, according to the protocol, no statistical analysis of OS in the PD-L1 IC+ subgroup was allowed. Nevertheless, the PD-L1 IC+ subgroup showed an absolute survival difference of almost 10 months between the two study arms (25.0 months vs. 15.5 months; HR 0.62; 95\%CI 0.45-0.86).

At SABC 2018 a more detailed biomarker-analysis beyond PD-L1 was presented. First of all, in this TNBC cohort, a much higher prevalence of PD-L1 expression on immune cells was observed compared to PDL1 expression on tumor cells (41\% vs. 9\%). In addition, these two subgroups were largely overlapping. Staining of PD-L1 was done by using the VENTANA SP142 IHC assay, which was approved by the FDA as companion diagnostic required for assessment of PD-L1 status in TBNC. A number of pathologists believe this biomarker evaluation to be of questionable value. The known weak staining capacity of SP142 for tumor cells opens the discussion if not other antibodies, more established for PD-L1 determination in other tumor entities, could be used as well [3].

For the PD-L1 IC-negative subgroup, absolutely no benefit by the addition of atezolizumab to nab-paclitaxel was shown both regarding PFS and OS. Within the PD-L1 positive subgroup, however, no difference between low (IC1; PFS HR 0.59; 95\%CI 0.44-0.78) and intermediate or strong staining (IC2/3; PFS HR 0.64; 95\%CI 0.43-0.97) was observed.

Beyond PD-L1 on immune cells, stromal tumorinfiltrating lymphocytes (sTILs) and tumor-infiltrating cytotoxic T cells (CD8+) were investigated as potential predictive markers. These markers by themselves, however, predicted clinical benefit with atezolizumab + nab-paclitaxel only if the infiltrating immune cells were also PD-L1 positive. Importantly, the efficacy of the combination treatment in PD-L1 IC+ patients was independent of their BRCA 1/2 mutation status.

Based on the study, atezolizumab received accelerated approval by the FDA in combination with nabpaclitaxel for the treatment of patients with triple-negative MBC with PD-L1 positivity on tumor-infiltrating immune cells $(\geq 1 \%)$.

\section{SOLAR-1: subgroup analyses}

A subgroup analysis of the SOLAR-1 data was performed by Juric et al. [4]. Phosphatidylinositol-3-kinase (PI3K) mutations are present in approximately $40 \%$ of patients with HR+/HER2- breast cancer leading to hyperactivation of the PI3K pathway and estrogen independent tumor growth $[5,6]$.
SOLAR-1 was a randomized, double-blind phase 3 study that investigated the efficacy and safety of the alpha-specific PI3K inhibitor alpelisib in combination with fulvestrant in patients with HR+/HER2- MBC. Patients were randomly assigned to receive alpelisib (300 mg/day p.o.) or placebo both in combination with fulvestrant (500 mg every 28 days and on days 1 and 15 of the first cycle i.m.). The primary results were presented at the 2018 ESMO congress. The study met its primary endpoint by nearly doubling median PFS in the PIK3CA-mutated subgroup (11 months vs 5.7 months; HR 0.65; 95\%CI 0.50-0.85; $P<0.001$ ). No benefit was seen in the PI3K-nonmutant cohort.

Three important subgroup analyses were presented in San Antonio: (1) Patients with prior CDK4/6 inhibitor therapy seemed to benefit in the same way from the PI3K inhibitor; however the subgroup was very small (18 patients) and the median duration of PFS in this subpopulation was short (5.5 vs 1.8 months; HR 0.48; 95\%CI 0.17-1.36). (2) The benefit with alpelisib was consistent in first- (HR 0.71) and second-line (HR 0.61). (3) PIK3CA mutation status assessed in plasma circulating tumor DNA (ctDNA) was of predictive value, similar or even better than the tumor tissue mutation status (HR 0.55; 95\%CI 0.39-0.79). However, the frequency of PIK3CA mutations in the plasma was much lower than in the tumor (34\% vs. $60 \%$ ), reflecting the detection limits of this technique.

OS data were still immature since only 92 deaths had occurred (52\% of the planned analysis): 40 in the alpelisib plus fulvestrant arm and 52 in the placebo plus fulvestrant arm (HR 0.73; 95\%CI 0.48-1.10; $P=0.06)$.

With $76 \%$ of patients having a grade $3 / 4$ adverse event, the treatment with alpelisib requires careful management of side effects. Most frequent all-grade adverse events were hyperglycemia $(65 \%$ vs $9 \%)$, diarrhea (54\% vs $11 \%$ ), nausea ( $46 \%$ vs $20 \%$ ) and rash ( $40 \%$ vs $6 \%$ ). Hyperglycemia is a reversible, on-target effect of PI3K inhibition, which is easily detectable and usually well manageable [7, 8]. However, in SOLAR-1, 44\% of patients with hyperglycemia had dose reductions and $87 \%$ received anti-diabetic medication (76\% with metformin). The risk for hyperglycemia was higher in patients who were prediabetic at baseline.

In conclusion, the combination of alpelisib and fulvestrant is a potential new treatment option for PIK3CA mutant HR+/HER2- MBC after progression on prior endocrine therapy. Given the challenging toxicity profile and the fact that the combination of everolimus and exemestane showed very similar results in BOLERO-2, irrespective of PI3K mutation status (HR $0.45 ; 95 \% 0.38-0.54 ; P<0.001$ ), the integration of this new treatment option in the therapeutic algorithm is not yet clear. 
CCTG MA38: randomized phase II study comparing two different schedules of palbociclib

The CDK 4/6 inhibitor palbociclib is approved by EMA and FDA at a standard dose of $125 \mathrm{mg}$ p.o. daily, 3 weeks on followed by one week off. Although this regimen is feasible for many patients, the on-off regimen might facilitate overdosing or non-adherence.

The Canadian Cancer Trials Group (CCTG) led a randomized phase II trial comparing the efficacy and tolerability of a continuous daily dose of $100 \mathrm{mg}$ p.o. daily (CDD) to $125 \mathrm{mg}$ p.o. daily 3 weeks on/1 week off (STD) [9]. Patients with HR+/HER2MBC after progression on first-line endocrine therapy were included. The primary endpoint was PFS, secondary endpoints included OS, safety and quality of life. In total 180 patients were enrolled. Palbociclib was found active in either $100 \mathrm{mg} \mathrm{CDD}$ or $125 \mathrm{mg}$ STD schedule: PFS in CDD was 9.3 months $(90 \%$ CI 6.93-13.9) compared to STD 11.3 months (90\%CI 8.08-13.83). However, while non-hematological toxicity profiles were comparable between both arms, more hematological adverse events were seen in the CDD arm. The rate of dose modification in CDD vs STD due to neutropenia was $70 \%$ vs $40 \%$, grade $3 / 4$ neutropenia $69 \%$ vs $53 \%$. The rate of febrile neutropenia was not different between the two arms. However, the higher rates of neutropenia and dose modifications do not favor CDD over STD in its current form and should therefore not be used in routine practice.

\section{Phase 1b trial of the BCL-2 inhibitor venetoclax combined with tamoxifen}

The B-cell lymphoma protein 2 (BCL-2) is an estrogen-responsive gene that is overexpressed in $80 \%$ of ER-positive breast cancers and associated with good prognosis in luminal A tumors [11-13]. Venetoclax is a potent inhibitor of BCL-2, routinely used in the treatment of chronic lymphatic leukemia (CLL). Its value in the treatment of solid tumors is yet to be evaluated. Preclinical data using xenograft models from ER+ breast cancer suggest that venetoclax shows a synergistic effect with tamoxifen to induce apoptosis [14]. Based on these findings, a phase $1 \mathrm{~b} 3+3$ doseescalation and expansion study was performed and presented in San Antonio [10]. The primary aim was to determine the maximum tolerated dose (MTD), define dose-limiting toxicities (DLTs) and to identify the recommended phase 2 dose (RP2D).

Thirty-three patients with ER+ (>1\%), BCL-2+ (>10\%, 2-3+ intensity), HER2- MBC received 200, 400,600 or $800 \mathrm{mg} /$ day of venetoclax together with tamoxifen $20 \mathrm{mg} /$ day. The MTD was not reached and the treatment was well tolerated with no DLT or high grade $3 / 4$ adverse events being observed. As a RP2D dose $800 \mathrm{mg} /$ day were selected and the cohort expanded to include 24 patients (15 patients had received prior therapies for $\mathrm{MBC}, 30 \%$ of them tamoxifen, $61 \%$ of patients had visceral metastases).

The overall response rate for the RP2D cohort $(n=24)$ included 1 complete response (CR $4 \%$ ) and 12 partial remissions (PR 50\%). The clinical benefit rate (CBR) was $75 \%$. The median duration of response were 42 weeks. Grade 3/4 adverse events included lymphopenia (6\% G3), neutropenia (6\% G3), anemia (6\% G3), diarrhea (6\% G3) and infections (9\% G3).

Based on these promising results a randomized phase 2 trial examining the combination of fulvestrant \pm venetoclax in patients with $\mathrm{HR}+/ \mathrm{HER}$ - disease after progression on CDK4/6 inhibitors was started (NCT03584009). Furthermore, the combination of venetoclax with palbociclib and letrozole is investigated in a phase Ib trial (PALVEN; NCT03900884).

\section{Conclusion}

Although no new large phase III clinical studies in MBC were presented at SABCS 2018, the shown data help to identify patients that might benefit most from potential or already established treatment options. Furthermore, some early phase studies, like the phase $1 \mathrm{~b}$ study of the BCL-2 inhibitor venetoclax, paved the way for promising and maybe practice changing clinical trials.

Funding Open access funding provided by Paracelsus Medical University.

Conflict of interest Theresa Westphal: Speakers Honoraria: Novartis; Travel grants: Roche, Pfizer. Simon Peter Gampenrieder: Speakers Honoraria: Novartis, Roche, AstraZeneca; Advisory Boards: Roche, Novartis, Pfizer, AstraZeneca; Travel grants: Roche, Novartis, Pfizer; Research funding: Roche. Richard Greil: Speakers Honoraria: Novartis, Roche, AstraZeneca; Advisory Boards: Roche, Novartis, AstraZeneca; Travel grants: Roche, Novartis, AstraZeneca; Research funding: Novartis, Roche, AstraZeneca

Open Access This article is distributed under the terms of the Creative Commons Attribution 4.0 International License (http://creativecommons.org/licenses/by/4.0/), which permits unrestricted use, distribution, and reproduction in any medium, provided you give appropriate credit to the original author(s) and the source, provide a link to the Creative Commons license, and indicate if changes were made.

\section{References}

1. Emens LALS, Loi S, Rugo HS, Schneeweiss A, Diéras V, Iwata $\mathrm{H}$ et al. Abstract GS1-04: IMpassion130: Efficacy in immune biomarker subgroups from the global, randomized, double-blind, placebo-controlled, phase III study of atezolizumab + nab-paclitaxel in patients with treatmentnaïve, locally advanced or metastatic triple-negative breast cancer. Cancer Res. 2019. https://doi.org/10.1158/15387445.SABCS18-GS1-04

2. Abdollahpour-Alitappeh $M$, Lotfinia $M$, Bagheri N, Sineh Sepehr K, Habibi-Anbouhi M, Kobarfard F, et al. Trastuzumab-monomethyl auristatin E conjugate exhibits 
potent cytotoxic activity in vitro against HER2-positive human breast cancer. J Cell Physiol. 2019;234(3):2693-704.

3. Sun WY, Lee YK, Koo JS. Expression of PD-L1 in triple-negativebreastcancerbasedondifferentimmunohistochemical antibodies. JTransl Med. 2016;14(1):173.

4. Juric D, Ciruelos E, Rubovszky G, Campone M, Loibl S, Rugo H, et al. Abstract GS3-08: Alpelisib + fulvestrant for advanced breast cancer: subgroup analyses from the phase III SOLAR-1 trial. Cancer Res. 2019;79(4 Supplement):GS308.

5. Crowder RJ, Phommaly C, Tao Y, Hoog J, Luo J, Perou CM, et al. PIK3CA and PIK3CB inhibition produce synthetic lethality when combined with estrogen deprivation in estrogen receptor-positive breast cancer. Cancer Res. 2009;69(9):3955-62.

6. MayerIA,AbramsonVG, FormisanoL, BalkoJM,EstradaMV, Sanders ME, et al. A phase Ib study of alpelisib (BYL719), a PI3Kalpha-specific inhibitor, with letrozole in ER+/HER2-metastatic breast cancer. Clin Cancer Res. 2017;23(1):26-34.

7. Baselga J, Dent SF, Cortés J, Im Y-H, Diéras V, Harbeck N, et al. Phase III study of taselisib (GDC-0032) + fulvestrant (FULV) v FULV in patients (pts) with estrogen receptor (ER)-positive, PIK3CA-mutant (MUT), locally advanced or metastatic breast cancer (MBC): primary analysis from SANDPIPER. J Clin Oncol. 2018;36(18_suppl):LBA1006LBA.

8. Baselga J, Campone M, Piccart M, Burris HA 3rd, Rugo HS, Sahmoud T, etal. Everolimus in postmenopausal hormonereceptor-positive advanced breast cancer. N Engl J Med. 2012;366(6):520.

9. Parulekar W, Joy A, Gelmon K, Mates M, Desbiens C, Clemons M, et al. Abstract PD1-10: Randomized phase II study comparing two different schedules of palbociclib plus second line endocrine therapy in women with estrogen receptor positive, HER2 negative advanced/metastatic breast cancer: CCTG MA38 (NCT02630693). Cancer Res.
2019. https://doi.org/10.1158/1538-7445.SABCS18-PD110.

10. Lindeman G, Lok S, Whittle J, Siow Z, Bergin A, Dawson S-J, et al. Abstract PD1-06: A phase 1b dose-escalation and expansion study of the BCL-2 inhibitor venetoclax combined with tamoxifen in ER and BCL-2-positive metastatic breast cancer (MBC). Cancer Res. 2019;79(4 Supplement):PD106-PD1.

11. Hwang KT, Han W, Kim J, Moon HG, Oh S, Song YS, et al. Prognostic influence of BCL2 on molecular subtypes of breast cancer. J Breast Cancer. 2017;20(1):54-64.

12. Oakes SR, Vaillant F, Lim E, Lee L, Breslin K, Feleppa F, et al. Sensitization of BCL-2-expressing breast tumors to chemotherapyby the BH3 mimeticABT-737. ProcNatlAcad SciUSA. 2012;109(8):2766-71.

13. Merino D, LokSW, VisvaderJE, Lindeman GJ. TargetingBCL2 to enhance vulnerability to therapy in estrogen receptorpositive breast cancer. Oncogene. 2016;35(15):1877-87.

14. Vaillant F, Merino D, Lee L, Breslin K, Pal B, Ritchie ME, et al. Targeting BCL-2 with the BH3 mimetic ABT-199 in estrogen receptor-positive breast cancer. Cancer Cell. 2013;24(1):120-9.

Publisher's Note Springer Nature remains neutral with regard to jurisdictional claims in published maps and institutional affiliations.

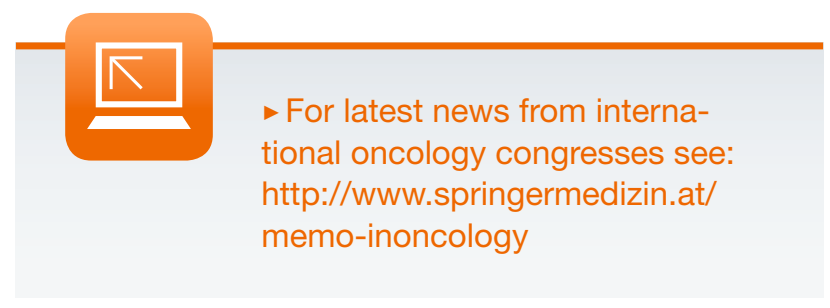

